\title{
Correlation of polymorphisms of the vascular endothelial growth factor gene and the risk of lung cancer in an ethnic Han group of North China
}

\author{
YAN LI ${ }^{1}$, JING LIANG ${ }^{1}$, XIAOLIN LIU $^{1}$, HAIRONG LIU $^{1}$, BEIBEI YIN ${ }^{1}$, JUNJUAN XIAO $^{1}$ and ZHENQIANG BI ${ }^{2}$ \\ ${ }^{1}$ Qianfoshan Hospital, Jinan, Shandong 250014; ${ }^{2}$ Shandong Center for Disease Control and Prevention, \\ Jinan, Shandong 250014, P.R. China
}

Received October 1, 2011; Accepted December 16, 2011

DOI: $10.3892 /$ etm.2012.453

\begin{abstract}
Vascular endothelial growth factor (VEGF) is a potent angiogenic mediator. The present study investigated the relationship between genetic polymorphisms of VEGF and susceptibility to lung cancer in a Han ethnic group of North China. The genotypes in the -2578C and 936C loci of VEGF gene were determined using PCR-RFLP method in 150 patients with lung cancers and 150 healthy individuals. Software PHASE 1.0 was used to analyze the experimental data. The non-conditional logistic regression model was used to analyze the statistical association of genotypes and susceptibility in the two groups adjusted by multiple factors. VEGF polymorphisms were found to be a critical risk for the genetic susceptibility to lung cancers in the ethnic Han group of North China. SNP polymorphisms at the $-2578 \mathrm{C}$ and 936C loci of the VEGF gene were detected by the RFLP-PCR method. High rates of a single-base C-to-A alteration at the -2578 locus and high rates of a single-base C-to-T alteration at the 936 locus of both alleles were correlated with the occurrence of lung cancer. The SNP markers at the $-2578 \mathrm{C}$ and 936C loci of the VEGF gene may serve as biological markers of lung cancer.
\end{abstract}

\section{Introduction}

Lung cancer is a major malignant tumor in China with an increasing incidence in most cities. In 2010, there were approximately 600,000 new cases of lung cancer in China. The majority of patients are already in the late stages when diagnosed, which significantly decreases the efficacy of chemotherapy and local radiotherapy. To improve the survival

Correspondence to: Dr Zhenqiang Bi, Shandong Center for Disease Control and Prevention, No. 16992 Jing Shi Road, Lixia, Jinan, Shandong 250014, P.R. China

E-mail: doctorlotus75@yahoo.cn

Key words: lung cancer, vascular endothelial growth factor gene, genetic polymorphism rates of lung cancer patients, accurate prognosis and early detection are critical. This will benefit public health and increase the efficacy of curing lung cancer.

Vascular endothelial growth factor (VEGF) is one of the main regulators of angiogenesis, that is, the formation of new blood vessels. VEGF works through VEGF-receptors (VEGFR) (1). Due to its predominant role in tumor angiogenesis, the VEGF-VEGFR system has been a suitable target for anticancer therapy (2). The normal function of VEGF is to produce new blood vessels (3). However, when VEGF is overexpressed, it largely contributes to tumor growth by stimulating blood vessel production. Therefore, regulation of VEGF expression is important and may be used for the prognosis of cancer, including lung cancers (4-7). In cells, gene expression is regulated by cellular transcriptional activators and inhibitors through protein-DNA binding with the promoter and promoter-regulating regions, which may be related to tumor angiogenesis and patient survival $(8,9)$.

DNA sequence polymorphisms, such as restriction fragment length polymorphisms and short tandem repeats, have been studied extensively in order to obtain useful biomarkers of diseases. However, single-nucleotide polymorphisms (SNPs) are another type of genetic markers, which have been studied in recent years (10-14). SNPs will aid in the understanding of the functions of human genes and have a significant impact on the diagnosis of the causes, treatment and prevention of human genetic diseases (11-14).

In the present study, SNP polymorphisms at the $-2578 \mathrm{C}$ and 936C loci of the VEGF gene were detected using the RFLP-PCR method. We found that high rates of a single-base C-to-A alteration at the -2578 locus and high rates of a singlebase C-to-T alteration at the 936 locus of both alleles were correlated with the occurrence of lung cancer. The SNP markers at the $-2578 \mathrm{C}$ and $936 \mathrm{C}$ loci of the VEGF gene may serve as biological markers of lung cancer.

\section{Materials and methods}

Patients. One hundred and fifty patients with primary lung cancerswere recruited in this study. The number of patients in stage I, II, III and IV was 5, 15, 60 and 70, respectively. The control group consisted of 150 healthy individuals. The 
age and gender distribution between the cancer group and the healthy controls had no significant difference $(\mathrm{P}>0.05)$.

DNA preparation. Peripheral blood mononuclear cells were isolated from the blood samples of patients by centrifugation, washed with buffers twice and stored at $-80^{\circ} \mathrm{C}$ for further analyses. The total DNAs were isolated and the purity of total DNAs was assessed by a UV240 spectrophotometer (Daojin Co., Japan).

Polymerase chain reaction (PCR). PCR was performed using a PCR machine (PE-2400; Perkin Elmer Cetus, USA) in a $100-\mu 1$ volume, containing $10 \mu \mathrm{l}$ of the cDNA samples, $10 \mu 1$ of the PCR buffer, 2 units of Taq DNA polymerase (Promega, USA), $125 \mathrm{nM}$ primer, $250 \mu \mathrm{M}$ each deoxyribonucleoside triphosphate (Shenggong Bio Co., Shanghai, China) and $2.5 \mathrm{mM}$ of $\mathrm{MgCl}_{2}$. The primers used are listed in Table I. The PCR products were separated on agarose gels. The density of the product band was analyzed by a gel analysis system (Chemi Imager 5500; Kadak, USA) using 1D Image Analysis Software 7 (Kadak).

Restriction fragment length polymorphism (RFLP). PCR products were digested with BglII, NlaIII at the -2578 and 936 loci of the VEGF gene, respectively. The enzyme digested products were separated on $1 \%$ agarose gels to obtain genotypes.

Statistical analyses. Data analyses were performed with SPSS (version 10.0) statistical software. The gender and age differences among the groups were not statistically significant. Data were analyzed using the $\chi^{2}$-test or the exact 4 -fold table method. The data measurements were conducted using a paired t-test or independent sample t-test. Data with differences having a P-values $<0.05$ were considered statistically significant.

\section{Results}

Detection of SNP polymorphisms at the -2578C locus of the $V E G F$ gene. To investigate the SNP polymorphisms at the -2578 locus of the VEGF gene, total DNAs were isolated from 150 lung cancer patients (lung cancer group) and 150 healthy individuals (control group), and PCR was performed. There were no statistically significant differences in age and gender distribution between the two groups $(\mathrm{P}>0.05)$. The PCR produced fragments of $\sim 400 \mathrm{bp}$ were as expected (data not shown). The PCR products were then subjected to BglII digestion to investigate whether a C-to-A single-base alteration occurred at the -2578 locus. As shown in Fig. 1A, if a C-to-A single-base alteration occurred at the -2578 locus, a new BglII locus was generated. As shown in Fig. 1C, digestion of the 400-bp fragments from the 300 individuals in both groups with $B g l$ II generated three types of band patterns on agarose gels: i) a band of 400 bp (lane 1), ii) two bands with sizes of $\sim 150$ and $250 \mathrm{bp}$, respectively, or iii) three bands with sizes of 150, 250 and $400 \mathrm{bp}$, respectively.

Cases with a RFLP pattern shown in lane 1 were considered wild-type (wt), with no base changes found at the $-2578 \mathrm{C}$ locus. Since $B g l$ II recognizes AGATCT, generation of the 150 and 250-bp bands may be explained by a single-base C-to-A alteration occurring at the -2578 locus, which generates a $B g l \mathrm{II}$
Table I. PCR primers and restricted enzymes.

\begin{tabular}{ll}
\hline Primers & \multicolumn{1}{c}{ Sequences } \\
\hline $2578 \mathrm{~F}$ & 5'-CTCCACCAAACCACAGCAAC-3' \\
$2578 \mathrm{R}$ & 5'-GGCTACTTCTCCAGGCTCAC-3' \\
$936 \mathrm{~F}$ & 5'-ACCACACCATCACCATCG-3' \\
$936 \mathrm{R}$ & 5'-CCAACTCAAGTCCACAGC-3' \\
\hline
\end{tabular}

locus. Since two bands with sizes of $\sim 150$ and $250 \mathrm{bp}$, but not the undigested (400 bp) bands, were found in lane 2, cases with a RFLP pattern shown in lane 2 may have a single-base C-to-A alteration at the -2578 locus of both alleles. However, in lane 3 , in addition to the 150- and 250-bp bands, the 400-bp bands were also found, suggesting that these cases may have a single-base C-to-A alteration at the -2578 locus of one of both alleles (Fig. 1B). The RFLP results suggest that the three types of SNP patterns are found at the -2578 locus of the VEGF gene. These three polymorphisms, including no alteration, a single-base C-to-A alteration at the -2578 locus of both alleles, a single-base C-to-A alteration at the -2578 locus of one of both alleles, were named wt, $\mathrm{C}-\mathrm{A} / \mathrm{C}-\mathrm{A}$ and $\mathrm{C}-\mathrm{A}$, respectively.

Detection of SNP polymorphisms at the 936C locus of the VEGF gene. The SNPs in the 936C locus of the VEGF gene were also determined for the 150 lung cancer patients (lung cancer group) and the 150 healthy individuals (control group) using the PCR and RFLP experimental methods. PCR produced fragments of $\sim 400 \mathrm{bp}$, as expected (data not shown). The PCR products were then subjected to NlaIII digestion to investigate whether a C-to-T single-base alteration occurred at the 936C locus. As shown in Fig. 2A, whether a C-to-T singlebase alteration occurred at the $936 \mathrm{C}$ locus, a new NlaIII locus was generated. As shown in Fig. 2C, digestion of the 400-bp fragments from the 300 individuals in both groups with NlaIII generated types of band patterns on agarose gels: i) a band of $\sim 400$ bp (lane 1), ii) two bands with sizes of $\sim 150$ and $250 \mathrm{bp}$, respectively, or iii) three bands with sizes of 150,250 and $400 \mathrm{bp}$, respectively.

Since NlaIII recognizes CATG, generation of the 150 - and 250-bp bands may be explained by a single-base C-to-T alteration at the 936C locus, which generates an NlaIII locus. Cases with a RFLP pattern as shown in lane 1 were found with only the 400-bp RFLP band, therefore, these cases were considered wt, since no base changes were found at the 936C locus. Since two bands with sizes of $\sim 150$ and $250 \mathrm{bp}$, but not the undigested (400 bp) bands, were found in lane 2, it suggested that cases with the RFLP bands shown in lane 2 may have a single-base C-to-T alteration at the 936C locus of both alleles. In lane 3 , in addition to the 150- and 250-bp bands, the 400-bp bands were also noted, suggesting that these cases may have a single-base C-to-A alteration at the 936C locus of one of both alleles (Fig. 2B). Altogether, the above RFLP results suggest that three types of SNP patterns are found at the 936C locus of the VEGF gene. These three polymorphisms, including no alteration, a single-base C-to-T alteration at the $-936 \mathrm{C}$ locus of both alleles, a single-base C-to-T alteration at the 936C 
A

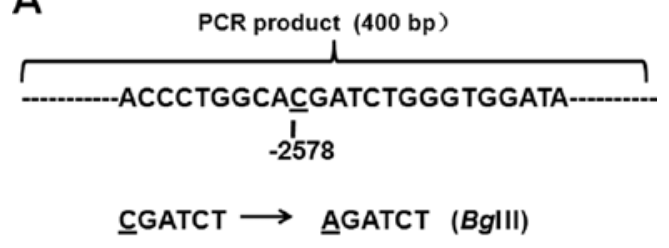

B

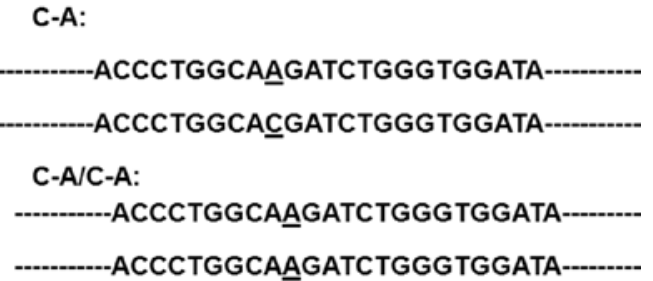

C

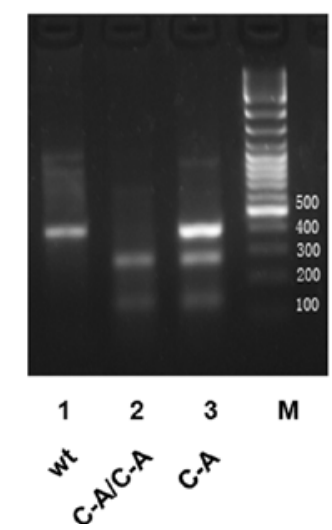

Figure 1. SNP polymorphisms at the $-2578 \mathrm{C}$ locus of the VEGF gene. (A) A putative single-base C-to-A alteration at the -2578 locus of the VEGF gene generated an additional $B g l \mathrm{II}$ site. (B) The single-base C-to-A alteration may occur at the -2578 locus of one allele (termed as C-A) or both alleles (termed as C-A/C-A). (C) SNP polymorphisms at $-2578 \mathrm{C}$ locus of the VEGF gene as detected by RFLP. Electrophoresis was performed. The experiments were performed more than three times. M, DNA marker (100-, 200-, 300-, 400-, 500-, 600-, 700- and 800-bp ladders).

Table II. Relationship between SNP polymorphisms at the $-2578 \mathrm{C}$ and $936 \mathrm{C}$ loci of the VEGF gene and the risk of lung cancer.

\begin{tabular}{lcccc}
\hline Sites & $\begin{array}{c}\text { Control } \\
\text { group }^{\mathrm{a}}\end{array}$ & $\begin{array}{c}\text { Lung cancer } \\
\text { group }^{\mathrm{a}}\end{array}$ & P-value & OR $(95 \% \mathrm{CI})$ \\
\hline
\end{tabular}

\begin{tabular}{lrrrl}
\hline$-2578 \mathrm{C}$ & & & & \\
wt & 98 & 93 & & \\
C-A & 49 & 45 & 0.003 & $0.412(0.287-0.674)$ \\
C-A/C-A & 3 & 12 & 0.059 & $3.212(0.947-11.895)$ \\
& & & & \\
936C & & & & \\
wt & 114 & 108 & & \\
C-T & 33 & 35 & 0.213 & $1.681(0.901-2.896)$ \\
C-T/C-T & 3 & 7 & 0.289 & $2.651(0.532-9.872)$ \\
\hline
\end{tabular}

${ }^{a}$ For both groups, $\mathrm{n}=150$. ${ }^{\mathrm{b}}$ The non-conditional logistic regression analysis was adjusted using multiple factors, including gender, smoking or not, and age. The logistic regression models were obtained using the wt genotype as the control group.
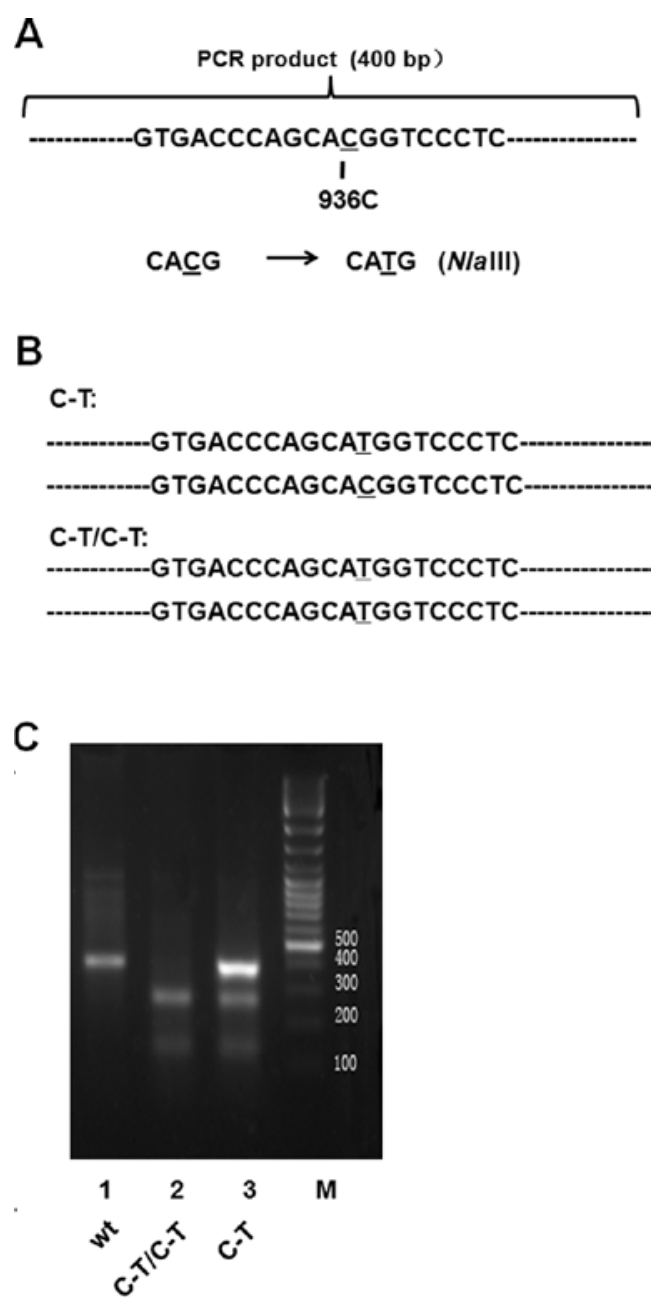

Figure 2. SNP polymorphisms at the 936C locus of the VEGF gene. (A) A putative single-base C-to-T alteration at the 936 locus of the VEGF gene generated an additional NlaIII site. (B) The single-base C-to-T alteration may occur at the 936 locus of one allele (termed as C-T) or both alleles (termed as C-T/C-T). (C) SNP polymorphisms at 936C locus of the VEGF gene as detected by RFLP. Electrophoresis was performed. The experiments were performed more than three times. M, DNA marker (100-, 200-, 300-, 400-, 500-, 600-, 700- and 800-bp ladders).

locus of one of both alleles, were named wt, C-T/C-T and C-T, respectively.

High rates of single-base C-to-A alteration at the -2578 locus and high rates of single-base C-to-T alteration at the 936 locus of both alleles correlate with occurrence of lung cancer. After identifying the SNPs of the 150 lung cancer cases and the 150 healthy individuals, we carried out statistical analyses of the SNPs at the $-2578 \mathrm{C}$ and $936 \mathrm{C}$ loci. It was found that the frequency distribution of the $-2578 \mathrm{C}$ and $936 \mathrm{C}$ SNPs at both alleles fits the Hardy-Weinberg equilibrium $(\mathrm{P}>0.05)$ (data not shown). Then, putative correlations between the genotype distribution of the $-2578 \mathrm{C}$ and 936C SNPs and the rate of lung cancer were investigated. As shown in Table II, most cases possessed a wt SNP at the $-2578 \mathrm{C}$ locus (113/150 and $98 / 150$ for the cancer group and the healthy control group, respectively). The two patterns with single-base alteration, $\mathrm{C}-\mathrm{A}$ and $\mathrm{C}-\mathrm{A} / \mathrm{C}-\mathrm{A}$, were found in cases of both groups. The control group and the cancer group had a similar number (49 
and 45, respectively) of C-A cases. The control group had $3 \mathrm{C}-\mathrm{A} / \mathrm{C}-\mathrm{A}$ cases, while the cancer group had $12 \mathrm{C}-\mathrm{A} / \mathrm{C}-\mathrm{A}$ cases. The increased numbers of C-A/C-A SNPs in the cancer group suggest that the homozygous C-A/C-A SNPs on both alleles may be a lung cancer-related disease marker.

Similar situations were also found for SNPs on the 936C loci. The healthy control group and the cancer group had similar numbers of wt or C-A SNPs (Table I). However, the healthy control group had $3 \mathrm{C}$-T/C-T cases, while the cancer group had 7 C-T/C-T cases. The increased numbers of C-T/C-T SNPs in the cancer group suggest that the homozygous C-T/C-T SNPs on both alleles may be related to the occurrence of lung cancer.

\section{Discussion}

SNP is defined as a DNA sequence polymorphism, caused by s variation in genome levels, including conversion, transferring, insertion and deletion of a single base. Theoretically, an SNP can be either a 2 allelic, 3 allelic or 4 allelic polymorphism. Among them, 3 or 4 allelic polymorphisms are not often found. Reported SNPs are commonly 2 allelic polymorphisms (10-14). The 2 allelic polymorphisms have four different types, i.e., $C \rightarrow T(G \rightarrow A), C \rightarrow A(G \rightarrow T), C \rightarrow G(G \rightarrow C)$ and $T \rightarrow A(A \rightarrow T)$. The frequency of the four types of SNPs occurring in humans varies. The most common type is $\mathrm{C} \rightarrow \mathrm{T}(\mathrm{G} \rightarrow \mathrm{A})$, accounting for approximately two-thirds of all SNPs. The frequency of the other three types is similar. The discovery of SNPs is conducted using gene sequencing, which helps discover diseases-related genes $(15,16)$. SNPs related to diseases mainly include the following types: virulence gene SNPs, diseases-susceptible SNPs, SNPs with diagnostic value and disease phenotype-associated SNPs. By analyzing SNP polymorphisms of patients and healthy individuals, it is possible to detect disease-susceptible genes or disease-related genes, which can provide a basis for exploring the pathogenesis, diagnosis and treatment of serious diseases.

By comparing and analyzing SNPs in one or more tumorsusceptible gene (including cis-acting element and splicing loci) of patients and healthy controls, differences in genotype distribution and SNP haplotype frequencies are determined. Therefore, susceptibility to a certain tumor as reflected by a genotype or haplotype can be predicted. Several studies have indicated the relationship between cancer susceptibility and VEGF gene polymorphisms. Renner et al (17) reported that the VEGF 936T allele was related to low levels of VEGF plasma and the decreased risk of breast cancer. VEGF expression was decreased by 3 -fold, possibly as the mutation of $936 \mathrm{C} \rightarrow \mathrm{T}$ caused the loss of the binding site of the transcription activator protein-4 (AP-4), finally resulting in low levels of VEGF blood plasma (17).

By analyzing the differences of SNP markers in cancer patients and healthy controls, the indicence rates and risks of cancers of various populations in a certain area can be evaluated. This is important for the prediction of cancers and following detection, the screening of cancer patients in early stages. In the present study, we found that VEGF polymorphisms may be associated with a risk for the genetic susceptibility to lung cancers in the ethnic Han group of North
China. SNP polymorphisms at the $-2578 \mathrm{C}$ and 936C loci of the VEGF gene were detected using the RFLP-PCR method. We found that high rates of a single-base C-to-A alteration at the -2578 locus and high rates of a single-base C-to-T alteration at the 936 locus of both alleles were correlated with the risk of lung cancer. SNP markers at the $-2578 \mathrm{C}$ and $936 \mathrm{C}$ loci of the VEGF gene may serve as biological markers of lung cancer.

\section{Acknowledgements}

This study was supported by the Natural Science Foundation of Shandong Province, China (grant no. 2005ZX04).

\section{References}

1. Shibuya M and Claesson-Welsh L: Signal transduction by VEGF receptors in regulation of angiogenesis and lymphangiogenesis. Exp Cell Res 312: 549-560, 2006.

2. Gotink KJ and Verheul HMW: Antiangiogenic tyrosine kinase inhibitors: What is their mechanism of action? Angiogenesis 13: $1-14,2010$.

3. O'Byrne KJ, Koukourakis MI, Giatromanolaki A, et al: Vascular endothelial growth factor, platelet-derived endothelial cell growth factor and angiogenesis in non-small cell cancer. Br J Cancer 82: 1427-1432, 2000.

4. Lee SJ, Lee SY, Jeon HS, et al: Vascular endothelial growth factor gene polymorphisms and risk of primary lung cancer. Cancer Epidemiol Biomarkers Prev 14: 571-575, 2005.

5. Baillie R, Harada K, Carlile J, Macluskey M, Schor SL and Schor AM: Expression of vascular endothelial growth factor in normal and tumor oral tissues with different antibodies. Histochem J 33: 287-294, 2001.

6. Salven P, Ruotsalainen T, Mattson K and Joensuu H: High pretreatment serum level of vascular endothelial growth factor (VEGF) is associated with poor outcome in small-cell lung cancer. Int J Cancer 79: 144-146, 2000.

7. Sheng H, Aoe M, Doihara H, Andou A and Shimizu N: Prognostic value of vascular endothelial growth factor expression in primary lung carcinoma. Acta Med Okayama 54: 119-126, 2000.

8. Yuan A, Yu CJ, Chen WJ, Lin FY, Kuo SH, Luh KT and Yang PC: Correlation of total VEGF mRNA and protein expression with histologic type, tumor angiogenesis, patient survival and timing of relapse in non-small cell lung cancer. Int J Cancer 89: 475-483, 2000.

9. Oshika Y, Nakamura M, Tokunaga T, et al: Expression of cellassociated isoform of vascular endothelial growth factor 189 and its prognostic relevance in non-small cell lung cancer. Int $\mathbf{J}$ Oncol 12: 541-544, 1998.

10. Collins FS, Patrinos A, Jordan E, Chakravarti A, Gesteland R and Walters L: New goals for the U.S. Human Genome Project: 1998-2003. Science 282: 682-689, 1998.

11. Wang DG, Fan JB, Siao CJ, et al: Large-scale identification, mapping, and genotyping of single-nucleotide polymorphisms in the human genome. Science 280: 1077-1082, 1998.

12. Roses AD: Pharmacogenetics and the practice of medicine. Nature 405: 857-865, 2000.

13. Kruglyak L and Nickerson DA: Variation is the spice of life. Nat Genet 27: 234-236, 2001.

14. Przeworski M, Hudson RR and di Rienzo A: Adjusting the focus on human variation. Trends Genet 16: 296-302, 2000.

15. Jacobs EJ, Feigelson HS, Bain EB, et al: Polymorphisms in the vascular endothelial growth factor gene and breast cancer in the Cancer Prevention Study II cohort. Breast Cancer Res 8: 22, 2006.

16. Jin Q,Hemminki K, Enquist K, et al: Vascular endothelial growth factor polymorphisms in relation to breast cancer development and prognosis. Clin Cancer Res 11: 3647-3653, 2005.

17. Renner W, Kotschan S, Hoffmann C, Obermayer-Pietsch B and Pilger E: A common $936 \mathrm{C} / \mathrm{T}$ mutation in the gene for vascular endothelial growth factor is associated with vascular endothelial growth factor plasma levels. J Vasc Res 37: 443-448, 2000. 